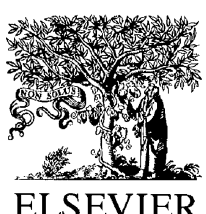

\title{
Rough set theory applied to (fuzzy) ideal theory
}

\author{
John N. Mordeson* \\ Department of Mathematics and Computer Science, Creighton University, 2500 California Plaza, Omaha, NE 68178, USA
}

Received 23 July 1998; received in revised form 8 December 1999; accepted 11 February 2000

\begin{abstract}
We use covers of the universal set to define approximation operators on the power set of the given set. In Section 1, we determine basic properties of the upper approximation operator and show how it can be used to give algebraic structural properties of certain subsets. We define a particular cover on the set of ideals of a commutative ring with identity in such a way that both the concepts of the (fuzzy) prime spectrum of a ring and rough set theory can simultaneously be brought to bear on the study of (fuzzy) ideals of a ring. (c) 2001 Elsevier Science B.V. All rights reserved.
\end{abstract}

Keywords: Algebra; Rough set; Cover; Approximation operator; Commutative ring; Ideal; Fuzzy ideal; Prime spectrum; Fuzzy prime spectrum

\section{Introduction}

In 1982, Pawlak introduced the concept of a rough set [18]. This concept is fundamental to the examination of granularity in knowledge. It is a concept which has many applications in data analysis. The idea is to approximate a subset of a universal set by a lower approximation and an upper approximation in the following manner. A partition of the universe is given. The lower approximation is the union of those members of the partition contained in the given subset and the upper approximation is the union of those members of the partition which have a nonempty intersection with the given subset. It is well known that a partition induces an equivalence relation on a set and vice versa. The properties of rough sets

\footnotetext{
* Tel.: +1-402-280-2418; fax: +1-402-280-5738.

E-mail address: mordes@creighton.edu (J.N. Mordeson).
}

can thus be examined via either partitions or equivalence relations. The members of the partition (or equivalence classes) can be formally described by unary set-theoretic operators [27], or by successor functions for upper approximation spaces $[7,8]$. This axiomatic approach allows not only for a wide range of areas in mathematics to fall under this approach, but also a wide range of areas to be used to describe rough sets. Some examples are topology, (fuzzy) abstract algebra, (fuzzy) directed graphs, (fuzzy) finite state machines, modal logic, interval structures $[7,14,15,17,19,27-29]$. One may generalize the use of partitions or equivalence relations to that of covers or relations [17,20,22,24,25,29].

In this paper, we use covers of the universal set to define approximation operators on the power set of the given set. In Section 1, we determine basic properties of the upper approximation operator and show how it can be used to give algebraic structural properties 
of certain subsets. Section 1 lays the ground work for our main results which appear in Section 2. In Section 2, we define a particular cover on the set of ideals of a commutative ring with identity in such a way that both the concepts of the (fuzzy) prime spectrum of a ring [6], and rough set theory can simultaneously be brought to bear on the study of (fuzzy) ideals of a ring. The notion of the (fuzzy) prime spectrum of a ring generalizes that of affine varieties, where the study of polynomial equations occurs. The notion of a fuzzy subset is of course due to Zadeh [30], and a fuzzy substructure of an algebraic structure is due to Rosenfeld [21].

\section{Upper and lower approximations defined by covers}

Let $V$ be nonempty set and let $\mathscr{P}(V)$ denote the power set of $V$. Let $s$ be a function of $\mathscr{P}(V)$ into itself. We are interested in the following conditions on $s$ since they are the ones that hold for upper approximation operators defined via an equivalence relation: (u1) $\forall X \in \mathscr{P}(V), X \subseteq s(X)$.

(u2) $\forall X, Y \in \mathscr{P}(V), X \subseteq Y \Rightarrow s(X) \subseteq s(Y)$.

(u3) $\forall X, Y \in \mathscr{P}(V), s(X \cup Y)=s(X) \cup s(Y)$.

(u4) $\forall X \in \mathscr{P}(V), s(X)=s(s(X))$.

Definition 1.1. Let $\mathscr{C} \subseteq \mathscr{P}(V) \backslash\{\emptyset\}$. Then $\mathscr{C}$ is called a cover of $V$ if $V \subseteq \bigcup_{C \in \mathscr{C}} C$. Suppose that $\mathscr{C}$ is a cover of $V$. Then

(i) $\mathscr{C}$ is said to be semi-reduced or semi-irredundant if $\forall C, D \in \mathscr{C}, C \subseteq D \Rightarrow C=D$;

(ii) $\mathscr{C}$ is said to be reduced or irredundant if $\forall C \in \mathscr{C}$, there does not exist $\mathscr{C}^{\prime} \subseteq \mathscr{C} \backslash\{C\}$ such that $C \subseteq \bigcup_{D \in \mathscr{C}^{\prime}} D$.

Definition 1.2. Let $\mathscr{C}$ be a cover of $V$. Define $\bar{s}: \mathscr{P}(V) \rightarrow \mathscr{P}(V)$ by $\forall X \in \mathscr{P}(V), \bar{s}(X)=\{y \in V \mid \exists C$ $\in \mathscr{C}, y \in C$ and $C \cap X \neq \emptyset\}$. Then $\forall X \in \mathscr{P}(V), \bar{s}(X)$ is called an upper approximation of $X$ with respect to $\mathscr{C}$. An upper approximation $\bar{s}$ is said to be transitive if $\forall x, y, z \in V, x \in \bar{s}(\{y\})$ and $y \in \bar{s}(\{z\})$ imply $x \in \bar{s}(\{z\})$

Proposition 1.3. Let $\mathscr{C}$ be a cover of $V$. Then the following properties hold.

(1) $\forall x \in V, x \in \bar{s}(\{x\})$ ( $\bar{s}$ is reflexive);
(2) $\forall x, y \in V, x \in \bar{s}(\{y\}) \Rightarrow y \in \bar{s}(\{x\})$

( $\bar{s}$ is symmetric);

(3) $\forall x \in V, \forall C \in \mathscr{C}, C \cap\{x\} \neq \emptyset \Rightarrow C \subseteq \bar{s}(\{x\})$;

(4) (u1)-(u3) hold.

Proof. (1) Let $x \in V$. Since $\mathscr{C}$ is a cover of $V, \exists C \in \mathscr{C}$ such that $x \in C$. Hence $C \cap\{x\} \neq \emptyset$. Thus $x \in \bar{s}(\{x\})$ by the definition of $\bar{s}$.

(2) Suppose $x \in \bar{s}(\{y\})$. Then $\exists C \in \mathscr{C}$ such that $x \in C$ and $C \cap\{y\} \neq \emptyset$. Hence $y \in C$ and $C \cap\{x\} \neq \emptyset$. Thus $y \in \bar{s}(\{x\})$.

(3) Suppose $C \cap\{x\} \neq \emptyset$. Then $\forall y \in C, y \in \bar{s}(\{x\})$ since $C \cap\{x\} \neq \emptyset$. Hence $C \subseteq \bar{s}(\{x\})$.

(4) Let $x \in X$. Then $\exists C \in \mathscr{C}$ such that $x \in C$ and clearly $C \cap X \neq \emptyset$. Hence $x \in \bar{s}(X)$. Thus (u1) holds. For (u2), let $z \in \bar{s}(X)$. Then $\exists C \in \mathscr{C}$ such that $z \in C$ and $C \cap X \neq \emptyset$. Hence $C \cap Y \neq \emptyset$ and so $z \in \bar{s}(Y)$. Consider (u3). Now $z \in \bar{s}(X \cup Y) \Leftrightarrow \exists C \in \mathscr{C}$ such that $z \in C$ and $C \cap(X \cup Y) \neq \emptyset \Leftrightarrow \exists C \in \mathscr{C}$ such that $z \in C$ and $(C \cap X) \cup(C \cap Y) \neq \emptyset \Leftrightarrow$ either $z \in \bar{s}(X)$ or $z \in \bar{s}(Y) \Leftrightarrow z \in \bar{s}(X) \cup \bar{s}(Y)$.

We are also interested in the following two conditions on a function $s: \mathscr{P}(V) \rightarrow \mathscr{P}(V)$ :

(u5) $\forall X \in \mathscr{P}(V)$ and $x \in V, x \in s(X)$ implies $\exists$ a finite subset $X^{\prime} \subseteq X$ such that $x \in s\left(X^{\prime}\right)$.

(u6) $\forall\left\{X_{\alpha} \mid \alpha \in \Omega\right\} \subseteq \mathscr{P}(V)$, $s\left(\bigcup_{\alpha \in \Omega} X_{\alpha}\right)=\bigcup_{\alpha \in \Omega} s\left(X_{\alpha}\right)$.

Condition (u5) plays an important role in determining structure results for algebraic structures [32].

When $V$ is finite, condition (u3) plays a major role, but when $V$ is infinite we often need condition (u6). The argument in Proposition 1.3 which shows that condition (u3) holds for $\bar{s}$ is immediately adaptable to show that condition (u6) holds for $\bar{s}$.

Example 1.4. Let $V=\mathbb{N} \cup\{\infty\}$, where $\mathbb{N}$ denotes the positive integers. Define $s: \mathscr{P}(V) \rightarrow \mathscr{P}(V)$ as follows: $\forall X \in \mathscr{P}(V)$, if $X$ is infinite, then $s(X)=X \cup\{\infty\}$ and if $X$ is finite, then $s(X)=X$. Clearly $s$ satisfies conditions (u1) and (u2). We now show that $s$ satisfies (u3). Let $X, Y \in \mathscr{P}(V)$.

Suppose that $X$ and $Y$ are finite. Then $X \cup Y$ is finite and so $s(X \cup Y)=X \cup Y=s(X) \cup s(Y)$.

Suppose that either $X$ is infinite or $Y$ is infinite. Then $X \cup Y$ is infinite. Hence $s(X \cup Y)=X \cup Y \cup\{\infty\}=$ $s(X) \cup s(Y)$. We now show that $s$ satisfies condition (u4). Let $X \in \mathscr{P}(V)$. Suppose that $X$ is finite. 
Then $s(X)=X$ and so $s(s(X))=s(X)=X=s(X)$. Suppose $X$ is infinite. Then $s(X)=X \cup\{\infty\}$ and so $s(s(X))=s(X \cup\{\infty\})=X \cup\{\infty\}=s(X)$. We now show that $s$ does not satisfy conditions (u5) and (u6). Now $\infty \in s(\mathbb{N})$, but $\nexists$ a finite subset $X^{\prime} \subseteq \mathbb{N}$ such that $\infty \in s\left(X^{\prime}\right)$ and so condition (u5) does not hold. Now $s\left(\bigcup_{n \in \mathbb{N}}\{n\}\right)=s(\mathbb{N})=V \neq \mathbb{N}=\bigcup_{n \in \mathbb{N}}\{n\}$ $=\bigcup_{n \in \mathbb{N}} s(\{n\})$. Hence condition (u6) does not hold.

Example 1.4 leads us to the following result.

Theorem 1.5. Suppose that $s: \mathscr{P}(V) \rightarrow \mathscr{P}(V)$ is such that (u2) holds. Then conditions (u3) and (u5) hold if and only if condition (u6) holds.

Proof. Suppose that (u3) and (u5) hold. Let $\left\{X_{\alpha} \mid\right.$ $\alpha \in \Omega\}$ be any subset of $\mathscr{P}(V)$. By (u2), $s\left(\bigcup_{\alpha \in \Omega} X_{\alpha}\right)$ $\supseteq s\left(X_{\alpha}\right) \forall \alpha \in \Omega$ and so $s\left(\bigcup_{\alpha \in \Omega} X_{\alpha}\right) \supseteq \bigcup_{\alpha \in \Omega} s\left(X_{\alpha}\right)$. Let $x \in s\left(\bigcup_{\alpha \in \Omega} X_{\alpha}\right)$. By (u5), $\exists$ finite subset $X^{\prime}$ of $\bigcup_{\alpha \in \Omega} X_{\alpha}$ such that $x \in s\left(X^{\prime}\right)$. Now $X^{\prime} \subseteq X_{\alpha_{1}} \cup \cdots \cup X_{\alpha_{n}}$ for some $\alpha_{1}, \ldots, \alpha_{n} \in \Omega$. Thus $x \in s\left(X_{\alpha_{1}} \cup \cdots \cup X_{\alpha_{n}}\right)$ $=s\left(X_{\alpha_{1}}\right) \cup \cdots \cup s\left(X_{\alpha_{n}}\right) \subseteq \bigcup_{\alpha \in \Omega} s\left(X_{\alpha}\right)$, where the equality holds by (u3). Hence $s\left(\bigcup_{\alpha \in \Omega} X_{\alpha}\right) \subseteq \bigcup_{\alpha \in \Omega} s\left(X_{\alpha}\right)$ and we have that (u6) holds. Conversely, suppose that (u6) holds. Let $X \in \mathscr{P}(V)$. Suppose that $y \in s(X)$. Then $y \in s\left(\bigcup_{x \in X}\{x\}\right)=\bigcup_{x \in X} s(\{x\})$. Thus $y \in s(\{x\})$ for some $x \in X$. Hence (u5) holds. It is immediate that (u3) holds.

Corollary 1.6. Let $\mathscr{C}$ be a cover of $V$. Suppose that condition (u4) holds for $\bar{s}$. Then $\forall X \in \mathscr{P}(V), \exists \mathscr{C}_{X} \subseteq$ $\mathscr{C}$ such that $\bar{s}(X)=\bigcup_{C \in \mathscr{C}_{X}} C$.

Proof. Let $x \in X$. Then $\forall C \in \mathscr{C}, C \cap\{x\} \neq \emptyset$ implies $C \subseteq \bar{s}(\{x\})$ by Proposition 1.3. Hence, $\bar{s}(\{x\})$ is the union of those $C \in \mathscr{C}$ such that $C \subseteq \bar{s}(\{x\})$. (Let $y \in \bar{s}(\{x\})$. Then $y \in C \in \mathscr{C}$ implies $C \subseteq \bar{s}(\{y\})$ $\subseteq \bar{s}(\bar{s}(\{x\}))=\bar{s}(\{x\})$ by (u4).) By (u6), $\bar{s}(X)=$ $\bigcup_{x \in X} \bar{s}(\{x\})$ from which the desired result holds.

Corollary 1.7. Let $\mathscr{C}$ be an irredundant cover of $V$. Suppose that condition (u4) holds for $\bar{s}$. Then $\forall x \in V, \forall C \in \mathscr{C}, C \cap\{x\} \neq \emptyset \Leftrightarrow C \subseteq \bar{s}(\{x\})$.

Proof. Let $x \in V$ and $C \in \mathscr{C}$. Suppose that $C \subseteq \bar{s}(\{x\})$. Then $C \subseteq \bar{s}(\{x\})=\bigcup\{D \in \mathscr{C} \mid x \in D\}$ by Corollary
1.4. If $C \cap\{x\}=\emptyset$, then $C \notin\{D \in \mathscr{C} \mid x \in D\}$ and we contradict the irredundancy of $\mathscr{C}$. Hence $C \cap\{x\} \neq \emptyset$. The converse follows by definition of $\bar{s}$.

Corollary 1.8 (Exchange property). Let $\mathscr{C}$ be a cover of $V$ and $X \subseteq V$. Then $\forall x, y \in V, x \in \bar{s}(X \cup\{y\})$ and $x \notin \bar{s}(X)$ implies $y \in \bar{s}(X \cup\{x\})$.

Proof. If $x \in \bar{s}(X \cup\{y\})$ and $x \notin \bar{s}(X)$, then $x \in \bar{s}(\{y\})$ by (u3). Hence $y \in \bar{s}(\{x\}) \subseteq \bar{s}(X \cup\{x\})$ by (2) of Proposition 1.3 and (u2).

The exchange property also plays an important role in determining structure results for algebraic structures [32].

Corollary 1.9. Let $\mathscr{C}$ be a cover of $V$. If condition (u4) holds for $\bar{s}$, then $\mathscr{P}=\{\bar{s}(\{x\}) \mid x \in V\}$ is a partition of $V$.

Proof. Since $x \in \bar{s}(\{x\}), V=\bigcup_{x \in V} \bar{s}(\{x\})$ by (u6). Suppose $\bar{s}(\{x\}) \cap \bar{s}(\{y\}) \neq \emptyset$. Let $z \in \bar{s}(\{x\}) \cap \bar{s}(\{y\})$. Then $\bar{s}(\{z\}) \subseteq \bar{s}(\bar{s}(\{x\})) \cap \bar{s}(\bar{s}(\{y\}))=\bar{s}(\{x\}) \cap$ $\bar{s}(\{y\})$. By (2) of Proposition 1.3, x,y $y \bar{s}(\{z\})$ and so $\bar{s}(\{x\}), \bar{s}(\{y\}) \subseteq \bar{s}(\{z\})$. Hence $\bar{s}(\{x\})=\bar{s}(\{z\})=$ $\bar{s}(\{y\})$. Thus $\mathscr{P}$ is a partition of $V$.

Example 1.10. Let $V=\{1,2,3\}$ and $\mathscr{C}=\left\{C_{1}, C_{2}\right\}$, where $C_{1}=\{1,2\}$ and $C_{2}=\{2,3\}$. Let $\bar{s}(\emptyset)=\emptyset$, $\bar{s}(\{1\})=\{1,2\}, \quad \bar{s}(\{3\})=\{2,3\}, \quad \bar{s}(\{2\})=V$, and $\bar{s}(X)=V$ if $X \in \mathscr{P}(V)$ and $|X|=2$, where $|X|$ denotes the cardinality of $X$. Thus $\bar{s}(\{1\})=\{1,2\} \subset V=\bar{s}$ $(\bar{s}(\{1\}))$. Hence property (u4) does not hold. Note also that $1 \in \bar{s}(\{2\}), 2 \in \bar{s}(\{3\})$, but $1 \notin \bar{s}(\{3\})$. Hence $\bar{s}$ is not transitive. We note that $\mathscr{C}$ is irredundant.

Example 1.11. Let $V=\{1,2\}$ and $\mathscr{C}=\{\{1\}, V\}$. Let $\bar{s}(\emptyset)=\emptyset$ and $\bar{s}(X)=V$ if $X \in \mathscr{P}(V)$ and $|X| \geqslant 1$. It follows easily that $\bar{s}(X)=\bar{s}(\bar{s}(X)) \quad \forall X \in \mathscr{P}(V)$. Also $\bar{s}$ is trivially transitive. However $\mathscr{C}$ is a cover of $V$, but not a partition of $V$. In fact, $\mathscr{C}$ is not semiirredundant.

Theorem 1.12. Let $\mathscr{C}$ be a cover of $V$. Then the following assertions are equivalent.

(1) $\mathscr{C} \quad$ is irredundant and $\forall X \in \mathscr{P}(V), \quad \bar{s}(X)=$ $\bar{s}(\bar{s}(X))$, i.e., (u4) holds. 
(2) $\mathscr{C}$ is a partition of $V$.

(3) $\mathscr{C}$ is irredundant and $\bar{s}$ is transitive.

Proof. (3) $\Rightarrow(2)$ : Let $C, D \in \mathscr{C}$ be distinct and suppose $C \cap D \neq \emptyset$. Let $x \in C \cap D$. Since $\mathscr{C}$ is irredundant, $C \backslash D \neq \emptyset \neq D \backslash C . \forall y \in C \backslash D$ and $\forall z \in D \backslash C, y \in \bar{s}(\{x\})$ since $C \cap\{x\} \neq \emptyset$ and $x \in \bar{s}(\{z\})$ since $D \cap\{z\} \neq \emptyset$. By the transitivity of $\bar{s}, y \in \bar{s}(\{z\})$. Hence there exists $C_{y, z} \in \mathscr{C}$ such that $y, z \in C_{y, z}$. Thus $(C \backslash D) \cup(D \backslash C) \subseteq$ $\bigcup\left\{C_{y, z} \mid y \in C \backslash D, z \in D \backslash C\right\}$ and $C \neq C_{y, z} \neq D$ for each pair $y \in C \backslash D, z \in D \backslash C$. Hence $C \subseteq\left(\bigcup\left\{C_{y, z} \mid y \in\right.\right.$ $C \backslash D, z \in D \backslash C\}) \cup D$ which contradicts the irredundancy of $\mathscr{C}$. Thus $C \cap D=\emptyset$ and so $\mathscr{C}$ is a partition of $V$.

$(2) \Rightarrow(1)$ : Let $X \in \mathscr{P}(V)$. Then $\bar{s}(X)=\bigcup_{C \in \mathscr{C}_{X}} C$, where $\mathscr{C}_{X}=\{C \in \mathscr{C} \mid C \subseteq \bar{s}(X)\}$ by Corollary 1.6. Thus

$$
\begin{aligned}
\bar{s}(\bar{s}(X)) & =\bar{s}\left(\bigcup_{C \in \mathscr{C}_{X}} C\right)=\bigcup_{C \in \mathscr{C}_{X}} \bar{s}(C) \\
& =\bigcup_{C \in \mathscr{C}_{X}} C=\bar{s}(X),
\end{aligned}
$$

where it remains to be shown that $\bar{s}(C)=C \forall C \in \mathscr{C}$. Let $y \in \bar{s}(C)$, where $C \in \mathscr{C}$. Then there exists $D \in \mathscr{C}$ such that $y \in D$ and $D \cap C \neq \emptyset$. Since $\mathscr{C}$ is a partition of $V, D=C$ and so $y \in C$. Hence $\bar{s}(C) \subseteq C$. That $C \subseteq \bar{s}(C)$ follows from Proposition 1.5.

$(1) \Rightarrow(3)$ : Suppose that $x \in \bar{s}(\{y\})$ and $y \in \bar{s}(\{z\})$. Then $x \in \bar{s}(\{y\}) \subseteq \bar{s}(\bar{s}(\{z\}))=\bar{s}(\{z\})$. Hence $\bar{s}$ is transitive.

In the next example, we show that (u4) can hold without $\mathscr{C}$ being a partition of $V$.

Example 1.13. Let $V=\{1,2,3\}$ and $\mathscr{C}=\{\{1,2\}$, $\{2,3\},\{1,3\}\}$. Let $\bar{s}(\emptyset)=\emptyset$ and $\bar{s}(X)=V \quad \forall X \in$ $\mathscr{P}(V), X \neq \emptyset$. Hence $\bar{s}$ satisfies (u4), $\bar{s}$ is transitive, and $\mathscr{C}$ is a semi-irredundant cover which is not a partition of $V . \mathscr{C}$ is not irredundant. (Note that $\{2,3\} \subseteq V=\bar{s}(\{1\})$ and $\{2,3\} \cap\{1\}=\emptyset$.)

Proposition 1.14. Let $\mathscr{C}$ be a cover of $V$. Then $\mathscr{C}$ is semi-irredundant and $\forall C \in \mathscr{C}, \bar{s}(C)=C$ if and only if $\mathscr{C}$ is a partition of $V$.

Proof. Suppose that $\mathscr{C}$ is semi-irredundant and $\forall C \in \mathscr{C}, \quad \bar{s}(C)=C$. Let $C, D \in \mathscr{C}$ be such that
$C \cap D \neq \emptyset$. Then $C=\bar{s}(C) \supseteq C \cup D$ by the definition of $\bar{s}$ and so $D \subseteq C$. Since $\mathscr{C}$ is semi-irredundant $D=C$. Thus $\mathscr{C}$ is a partition of $V$. The converse is immediate.

Let $s$ be a function of $\mathscr{P}(V)$ into itself. We are now interested in the following conditions on $s$ since they are the ones that hold for a lower approximation operator defined via an equivalence relation:

(11) $\forall X \in \mathscr{P}(V), X \supseteq s(X)$.

(12) $\forall X, Y \in \mathscr{P}(V), X \subseteq Y \Rightarrow s(X) \subseteq s(Y)$.

(13) $\forall X, Y \in \mathscr{P}(V), s(X \cap Y)=s(X) \cap s(Y)$.

(14) $\forall X \in \mathscr{P}(X), s(X)=s(s(X))$.

Definition 1.15. Let $\mathscr{C}$ be a cover of $V$. Define $\underline{s}$ : $\mathscr{P}(V) \rightarrow \mathscr{P}(V)$ by $\forall X \in \mathscr{P}(V), \underline{s}(X)=\{y \in V \mid \exists C \in$ $\mathscr{C}, y \in C$ and $C \subseteq X\}$. Then $\forall X \in \mathscr{P}(V), \underline{s}(X)$ is called a lower approximation of $X$ with respect to $\mathscr{C}$.

Proposition 1.16. Let $\mathscr{C}$ be a cover of $V$. Then $\forall X \in \mathscr{P}(V), \underline{s}(X) \supseteq V \backslash \bar{s}(V \backslash X)$.

Proof. Let $X \in \mathscr{P}(V)$. Then $\underline{s}(X)=\{y \in V \mid \exists C \in \mathscr{C}$, $y \in C, C \subseteq X\}=\bigcup\{C \mid C \in \mathscr{C}, C \subseteq X\}=\bigcup\{C \mid C \in$ $\mathscr{C}, C \cap(V \backslash X)=\emptyset\} \supseteq V \backslash \bigcup\{C \mid C \in \mathscr{C}, C \cap(V \backslash X) \neq$ $\emptyset\}=V \backslash \bar{s}(V \backslash X)$.

Proposition 1.17. Let $\mathscr{C}$ be a cover of $V$. Then $\forall X \in \mathscr{P}(V), V \backslash \underline{s}(X)=\bar{s}(V \backslash X)$ if and only if $\mathscr{C}$ is a partition of $V$.

Proof. Suppose that $\forall X \in \mathscr{P}(V), V \backslash \underline{s}(X)=\bar{s}(V \backslash X)$. If $\mathscr{C}=\{V\}$, then $\mathscr{C}$ is a partition of $V$. Suppose that $\mathscr{C} \neq\{V\}$. Then $\exists C_{1}, C_{2} \in \mathscr{C}$ such that $C_{1}$ $\nsubseteq C_{2}$. By definition of $\underline{s}, \underline{s}\left(C_{2}\right)=C_{2}$. Hence $\bar{s}\left(V \backslash C_{2}\right)$ $=V \backslash \underline{s}\left(C_{2}\right)=V \backslash C_{2}$. Since $C_{1} \nsubseteq C_{2}, C_{1} \subseteq \bar{s}\left(V \backslash C_{2}\right)$ $=V \backslash \underline{s}\left(C_{2}\right)=V \backslash C_{2}$. Hence $C_{1} \cap C_{2}=\emptyset$. Thus $\mathscr{C}$ is a partition of $V$. The converse is well known.

Proposition 1.18. Let $\mathscr{C}$ be a cover of $V$. Then the following assertions hold.

(1) $\underline{s}$ satisfies (11) and (12);

(2) $\forall X, Y \in \mathscr{P}(V), \underline{s}(X \cap Y) \subseteq \underline{s}(X) \cap \underline{s}(Y)$.

(3) $\forall X \in \mathscr{P}(V), \underline{s}(X) \supseteq \underline{s}(\underline{s}(X))$.

Proof. Let $X, Y \in \mathscr{P}(V)$ be such that $X \subseteq Y$. Suppose that $\underline{s}(X)=\emptyset$. Then clearly (11) and (12) hold. Suppose that $\underline{s}(X) \neq \emptyset$. Let $y \in \underline{s}(X)$. Then $\exists C \in \mathscr{C}$ such 
that $y \in C \subseteq X \subseteq Y$. Thus assertion (1) holds. That assertion (2) holds follows from assertion (1). That assertion (3) hold follows easily from assertion (1).

In Example 1.10, we have $\underline{s}\left(C_{1} \cap C_{2}\right)=\underline{s}(\{2\})=\emptyset$ and $\underline{s}\left(C_{1}\right) \cap \underline{s}\left(C_{2}\right)=C_{1} \cap C_{2}=\{2\}$. Hence strict inclusion in (2) of Proposition 1.18 is possible.

We note that if $y \in \bar{s}(X)$, then $\exists$ a finite subset $X^{\prime}$ of $X$ such that $y \in \bar{s}\left(X^{\prime}\right): y \in \bar{s}(X)$ implies $y \in \bigcup_{x \in X} \bar{s}(\{x\})$ by (u6) which in turn implies $y \in \bar{s}(\{x\})$ for some $x \in X$.

This result together with properties (u1), (u2), (u4), and Corollary 1.8 yield structural results similar to those of certain algebraic structures [8]. We list them below since they are also used in the next section.

Definition 1.19. Let $\mathscr{C}$ be a cover for $V$ and suppose that $\bar{s}$ satisfies (u4). Let $X$ be a subset of $V$.

(1) A subset $U$ of $V$ is called a subspace of $V$ if $U=\bar{s}(U)$.

(2) $X$ is called free if $\forall x \in X, x \notin \bar{s}(X \backslash\{x\})$.

(3) $X$ is said to generate a subspace $U$ of $V$ if $U=\bar{s}(X)$.

(4) $X$ is called a basis for a subspace $U$ of $V$ if $X$ generates $U$ and if $X$ is free.

Theorem 1.20 (Kuroki and Mordeson [8]). Let $\mathscr{C}$ be a cover for $V$ and suppose that $\bar{s}$ satisfies (u4). Let $X$ be a subset of $V$. Then the following properties are equivalent.

(1) $X$ is a minimal generating set of $V$.

(2) $X$ is a maximally free subset of $V$.

(3) $X$ is a basis of $V$.

Theorem 1.21 (Kuroki and Mordeson [8]). Let $\mathscr{C}$ be a cover for $V$ and suppose that $\bar{s}$ satisfies (u4). Then $V$ has a basis and the cardinality of a basis is unique.

As an example, consider Example 1.13. $\{1\}$ is a basis for $V$ as is $\{2\}$ and also $\{3\}$.

Properties (u1)- $(\mathrm{u} 4)$ yield structural results similar to those of (fuzzy) finite state machines and (fuzzy) directed graphs. These results deal with such concepts as primaries, connectedness, and retrievability [14] and [15]. The interested reader can easily find them in the references.

We now consider another approach. Let $\bar{g}, g$ : $\mathscr{P}(V) \rightarrow \mathscr{P}(V)$.
Clearly, $\forall X \in \mathscr{P}(V), \underline{g}(X)=V \backslash \bar{g}(V \backslash X) \Leftrightarrow \bar{g}(X)$ $=V \backslash \underline{g}(V \backslash X)$.

Proposition 1.22. Suppose that $\forall X \in \mathscr{P}(V), \bar{g}(X)=$ $V \backslash \underline{g}(V \backslash X)$. Then $\bar{g}$ satisfies (ui) $\Leftrightarrow \underline{g}$ satisfies (1i) for $i=1,2,3,4$.

Proof. Let $X, Y \in \mathscr{P}(V)$. Then $X \subseteq \bar{g}(X) \Leftrightarrow X \subseteq$ $V \backslash g(V \backslash X) \Leftrightarrow V \backslash X \supseteq g(V \backslash X)$. Hence $\bar{g}$ satisfies (u1) $\Leftrightarrow g$ satisfies (11). Suppose that $X \subseteq Y$. Then $\bar{g}(X) \subseteq \overline{\bar{g}}(Y) \Leftrightarrow V \backslash \underline{g}(V \backslash X) \subseteq V \backslash \underline{g}(V \backslash Y) \Leftrightarrow \underline{g}(V \backslash X)$ $\supseteq g(V \backslash Y)$. Hence $\bar{g}$ satisfies (u2) $\Leftrightarrow g$ satisfies (12). Now $\bar{g}(X \cup Y)=\bar{g}(X) \cup \bar{g}(Y) \Leftrightarrow V \backslash g \overline{(}(V \backslash X \cup Y)$ $=V \backslash g(V \backslash X) \cup V \backslash g(V \backslash Y) \Leftrightarrow g(V \backslash X \cup Y \overline{)}=g(V \backslash X)$ $\cap \underline{g}(\overline{V \backslash Y}) \Leftrightarrow \underline{g}((V \backslash \bar{X}) \cap(V \backslash Y \overline{)})=\underline{g}(V \backslash X) \cap \underline{g}(V \backslash Y)$. Hence $\bar{g}$ satisfies (u3) $\Leftrightarrow g$ satisfies (13). Now $\bar{g}(X)$ $=\bar{g}(\bar{g}(X)) \Leftrightarrow V \backslash \underline{g}(V \backslash X) \stackrel{\underline{g}}{=} V \backslash \underline{g}(V \backslash \bar{g}(X)) \Leftrightarrow \underline{g}(V \backslash X)$ $=g(V \backslash \bar{g}(X)) \Leftrightarrow g(V \backslash X)=\bar{g}(V \backslash \bar{g}(V \backslash g(V \backslash X))) \Leftrightarrow$ $\underline{g}(\bar{V} \backslash X)=\underline{g}(V \backslash(\overline{V \backslash} \underline{g}(V \backslash X))) \Leftrightarrow \underline{g}(V \backslash \bar{X})=\underline{g}(\underline{g}(V \backslash$ $\bar{X})$ ). Hence $\bar{g}$ satisfies (u4) $\Leftrightarrow \underline{g}$ satisfies (14).

Let $s: \mathscr{P}(V) \rightarrow \mathscr{P}(V)$ be such that (u1) holds. Define $\mathscr{C}=\{s(\{x\}) \mid x \in V\}$. We have $x \in s(\{x\})$. Thus $\mathscr{C}$ is a cover of $V$ called the cover induced by $s$.

Proposition 1.23. Let $\bar{g}, \underline{g}: \mathscr{P}(V) \rightarrow \mathscr{P}(V)$. Suppose that $x \in \bar{g}(\{x\}) \quad \forall x \in V$. Let $\mathscr{C}=\{\bar{g}(\{x\}) \mid x \in V\}$. Then any two of the following three assertions implies the third.

(1) $\forall X \in \mathscr{P}(V), \quad \underline{g}(X)=V \backslash \bar{g}(V \backslash X)$ and $\bar{g}(X)=$ $V \backslash g(V \backslash X)$.

(2) $\forall X \in \mathscr{P}(V), \bar{g}(X)=\{z \in V \mid \bar{g}(\{z\}) \cap X \neq \emptyset\}$.

(3) $\forall X \in \mathscr{P}(V), g(X)=\{z \in V \mid \bar{g}(\{z\}) \subseteq X\}$.

Proof. (2) and (3) $\Rightarrow$ (1): Let $X \in \mathscr{P}(V)$. Then $y \in V \backslash \bar{g}(V \backslash X) \Leftrightarrow y \notin \bar{g}(V \backslash X) \Leftrightarrow y \notin\{z \in V \mid \bar{g}(\{z\})$ $\cap(V \backslash X) \neq \emptyset\} \Leftrightarrow y \in\{z \in V \mid \bar{g}(\{z\}) \cap(V \backslash X)=\emptyset\}$ $\Leftrightarrow y \in\{z \in V \mid \bar{g}(\{z\}) \subseteq X\} \Leftrightarrow y \in g(X)$, where we note that $y \notin\{z \in V \mid \bar{g}(\{z\}) \cap(V \backslash X) \neq \emptyset\} \Leftrightarrow \bar{g}(\{y\})$ $\cap(V \backslash X)=\emptyset$. Thus $\quad \underline{g}(X)=V \backslash \bar{g}(V \backslash X)$ and so $\bar{g}(X)=V \backslash g(V \backslash X)$.

(1) and (3) $\Rightarrow$ (2): $y \in \bar{g}(X) \Leftrightarrow y \in V \backslash g(V \backslash X) \Leftrightarrow$ $y \notin \underline{g}(V \backslash X) \Leftrightarrow y \notin\{z \in V \mid \bar{g}(\{z\}) \subseteq V \backslash \bar{X}\} \Leftrightarrow y \in$ $\{z \in V \mid \bar{g}(\{z\}) \cap X \neq \emptyset\}$.

(1) and (2) $\Rightarrow(3): y \in g(X) \Leftrightarrow y \in V \backslash \bar{g}(V \backslash X) \Leftrightarrow$ $y \notin \bar{g}(V \backslash X) \Leftrightarrow y \notin\{z \in V \mid \bar{g}(\{z\}) \cap(V \backslash X) \neq \emptyset\} \Leftrightarrow$ $y \in\{z \in V \mid \bar{g}(\{z\}) \subseteq X\}$. 


\section{Upper and lower approximation applied to commutative rings}

Let $R$ be a commutative ring with identity. We use the notion of the prime spectrum of $R$ to define a cover of $\mathscr{I}=\{I \mid I$ is an ideal of $R, I \neq R\}$. Let $\mathscr{P}$ denote the set of all prime ideals of $R$ and $\forall X \in \mathscr{P}(\mathscr{I})$, the power set of $\mathscr{I}$, let $\mathscr{P}_{X}$ denote $\{P \in \mathscr{P} \mid \exists I \in X$ such that $P \supseteq I\}$. Then $\mathscr{P}$ is known as the prime spectrum of $R$ and the closed subsets of $\mathscr{P}$ in the Zariski topology are exactly the $\mathscr{P}_{\{I\}}$, where $I \in \mathscr{I}$. Let $\mathscr{C}=\left\{\mathscr{P}_{\{I\}} U\{I\} \mid I \in \mathscr{I}\right\}$. Then $\mathscr{C}$ is a cover of $\mathscr{I}$. Now $\mathscr{C}$ is not necessarily semi-irredundant. For example, let $R$ denote the polynomial ring $F[x, y]$ in algebraically independent indeterminates $x, y$ over the field $F$. Consider the prime ideals $\langle x\rangle$ and $\langle x, y\rangle$ of $R$. Then $\mathscr{P}_{\{\langle x, y\rangle\}} \cup\{\langle x, y\rangle\}=\mathscr{P}_{\{\langle x, y\rangle\}} \subset \mathscr{P}_{\{\langle x\rangle\}}=\mathscr{P}_{\{\langle x\rangle\}} \cup\{\langle x\rangle\}$.

Define $\bar{s}: \mathscr{P}(\mathscr{I}) \rightarrow \mathscr{P}(\mathscr{I})$ as in Definition 1.2, i.e., $\forall X \in \mathscr{P}(\mathscr{I}), \quad \bar{s}(X)=\left\{J \in \mathscr{I} \mid \exists \mathscr{P}_{\{I\}} \cup\{I\} \in \mathscr{C}, \quad J \in\right.$ $\mathscr{P}_{\{I\}} \cup\{I\}$ and $\left.\left(\mathscr{P}_{\{I\}} \cup\{I\}\right) \cap X \neq \emptyset\right\}$. Define $\bar{g}: \mathscr{P}(\mathscr{I}) \rightarrow \mathscr{P}(\mathscr{I})$ by $\forall X \in \mathscr{P}(\mathscr{I}), \quad \bar{g}(X)=\mathscr{P}_{X} \cup X$. Then $\mathscr{C}$ is the cover induced by $\bar{g}$. In fact, $\bar{g}$ here defines $\mathscr{C}$ just as does the $\bar{g}$ in Section 1 since $\mathscr{C}=\{\bar{g}(\{I\}) \mid I \in \mathscr{I}\}$.

By choosing the cover $\mathscr{C}$ in the above manner, we can apply techniques from both algebraic geometry and rough set theory to the study of the ideal theory: By adjoining $\{I\}$ to $\mathscr{P}_{\{I\}}$, we will be able to show that $\bar{g}$ satisfies (u1)-(u4).

Proposition 2.1. Let $R$ be a commutative ring with identity and let $\bar{s}$ and $\bar{g}$ be defined as above. Then $\forall X \in \mathscr{P}(I), \bar{g}(X) \subseteq \bar{s}(X)$.

Proof. Let $J \in \bar{g}(X)$. Then either $J \in \mathscr{P}_{X}$ or $J \in X$. Suppose $J \in X$. Then $J \in \mathscr{P}_{\{J\}} \cup\{J\}$ and $\left(\mathscr{P}_{\{J\}} \cup\{J\}\right)$ $\cap X \neq \emptyset$. Thus $J \in \bar{s}(X)$. Suppose $J \in \mathscr{P}_{X}$. Then $J$ is a prime ideal of $R$ and $\exists K \in X$ such that $J \supseteq K$. Hence $J \in \mathscr{P}_{\{K\}} \cup\{K\}$ and $\left(\mathscr{P}_{\{K\}} \cup\{K\}\right) \cap X \neq \emptyset$. Thus $J \in \bar{s}(X)$. Hence $\bar{g}(X) \subseteq \bar{s}(X)$.

Proposition 2.2. Let $R$ be a commutative ring with identity and let $\bar{s}$ and be defined as above.

(1) If $K$ is an ideal of $R$ which is not prime, then $\bar{s}(\{K\})=\mathscr{P}_{\{K\}} \cup\{K\}$.

(2) If $P$ is a prime ideal, then $\bar{s}(\{P\})=\mathscr{P} \cup S_{P}$, where $S_{P}=\{I \in \mathscr{I} \mid I \subseteq P\}$
Proof. (1) $\bar{s}(\{K\})=\left\{J \in \mathscr{I} \mid \exists \mathscr{P}_{\{I\}} \cup\{I\} \in \mathscr{C}, J \in\right.$ $\mathscr{P}_{\{I\}} \cup\{I\}$ and $\left.\left(\mathscr{P}_{\{I\}} \cup\{I\}\right) \cap\{K\} \neq \emptyset\right\}=\mathscr{P}_{\{K\}} \cup$ $\{K\}$ since $K$ is not prime and so $\left(\mathscr{P}_{\{I\}} \cup\{I\}\right) \cap$ $\{K\} \neq \emptyset$ if and only if $I=K$.

(2) $\bar{s}(\{P\})=\left\{J \in \mathscr{I} \mid \exists \mathscr{P}_{\{I\}} \cup\{I\} \in \mathscr{C}, J \in \mathscr{P}_{\{I\}}\right.$ $\cup\{I\}$ and $\left.\left(\mathscr{P}_{\{I\}} \cup\{I\}\right) \cap\{P\} \neq \emptyset\right\}$. Now $\left(\mathscr{P}_{\{I\}} \cup\right.$ $\{I\}) \cap\{P\} \neq \emptyset$ if and only if either $P \in \mathscr{P}_{\{I\}}$ or $P=I$. If $P \in \mathscr{P}_{\{I\}}$, then $J \in \mathscr{P}_{\{I\}} \cup\{I\}$ if and only if $J$ is either a prime ideal containing an ideal which $P$ contains or $J \subseteq I$, where $I \subseteq P$. If we let $I=\langle 0\rangle$, then $P \supseteq I$ and every $J \in \mathscr{P}$ contains $I$.

Example 2.3. Let $R$ denote the polynomial ring $F[x]$ in an indeterminate $x$ over a field $F$ and let $\bar{s}$ and be defined as above. We show that $\bar{s}\left(\left\{\left\langle x^{4}\right\rangle\right\}\right) \subset$ $\bar{s}\left(\bar{s}\left(\left\{\left\langle x^{4}\right\rangle\right\}\right)\right)$. By Proposition 2.2, $\bar{s}\left(\left\{\left\langle x^{4}\right\rangle\right\}\right)=\mathscr{P}_{\left\{\left\langle x^{4}\right\rangle\right\}}$ $\cup\left\{\left\langle x^{4}\right\rangle\right\}=\{\langle x\rangle\} \cup\left\{\left\langle x^{4}\right\rangle\right\}$. Now $\bar{s}\left(\bar{s}\left(\left\{\left\langle x^{4}\right\rangle\right\}\right)\right)=$ $\bar{s}\left(\{\langle x\rangle\} \cup\left\{\left\langle x^{4}\right\rangle\right\}\right)=\bar{s}(\{\langle x\rangle\}) \cup \bar{s}\left(\left\{\left\langle x^{4}\right\rangle\right\}\right)=\bar{s}(\{\langle x\rangle\}) \cup$ $\left(\{\langle x\rangle\} \cup\left\{\left\langle x^{4}\right\rangle\right\}\right)$. Now $\bar{s}(\{\langle x\rangle\})=\mathscr{P} \cup S_{\{\langle x\rangle\}}$. Therefore, we see that $\left\langle x^{2}\right\rangle \in \bar{s}\left(\bar{s}\left(\left\{\left\langle x^{4}\right\rangle\right\}\right)\right)$, but $\left\langle x^{2}\right\rangle \notin$ $\bar{s}\left(\left\{\left\langle x^{4}\right\rangle\right\}\right)$.

Example 2.4. Let $R$ denote the polynomial ring $F[x, y]$ in indeterminates $x$ and $y$ over a field $F$ and let $\bar{s}$ and be defined as above. Consider the prime ideal $P=\langle x, y\rangle$ of $R$. Let $p(y) \in R, p(y) \neq y$, be irreducible over $F$. Then $\langle x, p(y)\rangle \in \mathscr{P}$. Now $\langle x, p(y)\rangle \notin \mathscr{P}_{\{P\}}$ and $\langle x, p(y)\rangle \notin S_{P}$.

A commutative ring $R$ with identity is said to be quasi-local if it has a unique maximal ideal.

Proposition 2.5. Let $R$ be a quasi-local ring. Let $\bar{s}$ be defined as above. Let $M$ denote the unique maximal ideal of $R$. Then $\bar{s}(\{M\})=\mathscr{I}$.

Proof. $\bar{s}(\{M\})=\mathscr{P} \cup S_{\{M\}}=\mathscr{I}$ since $M$ contains every ideal of $R$.

Let $R$ denote $F_{1} \oplus F_{2}$, the direct sum of the fields $F_{1}$ and $F_{2}$. Then $\mathscr{I}=\{\langle(0,0)\rangle,\langle(0,1)\rangle,\langle(1,0)\rangle\}$. Then $M_{1}=\langle(1,0)\rangle$ and $M_{2}=\langle(0,1)\rangle$ are maximal ideals of $R$. Now $\bar{s}\left(\left\{M_{1}\right\}\right)=\bar{s}\left(\left\{M_{2}\right\}\right)=\bar{s}(\{\langle(0,0)\rangle\})=\mathscr{I}$, but $R$ is not quasi-local and $\langle(0,0)\rangle$ is not a prime ideal of $R$. We see that the converse of Proposition 2.5 does not hold.

Theorem 2.6. Let $R$ be a commutative ring with identity and let $\bar{g}$ be defined as above. Then $\bar{g}$ satisfies (u1)-(u4). 
Proof. By definition of $\bar{g}, X \subseteq \bar{g}(X) \forall X \in \mathscr{P}(\mathscr{I})$ and so (u1) holds. Suppose $X, Y \in \mathscr{P}(\mathscr{I})$ and $X \subseteq Y$. If $P$ is a prime ideal of $R$ for which there exists $I \in X$ such that $P \supseteq I$, then $P$ contains an ideal in $Y$, namely $I$. Thus $\bar{g}(X) \subseteq \bar{g}(Y)$ and so (u2) holds. For $X, Y \in \mathscr{P}(\mathscr{I}), \quad \bar{g}(X \cup Y)=\{P \in \mathscr{P} \mid \exists I \in X \cup Y$ such that $P \supseteq I\} \cup X \cup Y=\{P \in \mathscr{P} \mid \exists I \in X$ such that $P \supseteq I\} \cup X \cup\{P \in \mathscr{P} \mid \exists I \in Y$ such that $P \supseteq I\} \cup Y=$ $\bar{g}(X) \cup \bar{g}(X)$ and so (u3) holds. Let $X \in \mathscr{P}(\mathscr{I})$ and $P \in \bar{g}(\bar{g}(X))$. Then either $P$ is a prime ideal of $R$ such that $\exists Q \in \bar{g}(X)$ such that $P \supseteq Q$ or $P \in \bar{g}(X)$. Suppose $P$ is a prime ideal of $R$ such that $\exists Q \in \bar{g}(X)$ such that $P \supseteq Q$. Then either $Q$ is a prime ideal of $R$ such that $\exists J \in X$ such that $Q \supseteq J$ or $Q \in X$ and so $P \in \bar{g}(X)$ since $P \supseteq Q$ and $P$ is a prime ideal. In any of these cases, we have $P \in \bar{g}(X)$. Thus $\bar{g}(\bar{g}(X)) \subseteq \bar{g}(X)$. Hence (u4) holds since $\bar{g}(X) \subseteq \bar{g}(\bar{g}(X))$ by (s1).

Corollary 2.7. Let $R$ be a commutative ring with identity and let $\bar{g}$ be defined as above. Then $\bar{g}$ satisfies (u6).

Proof. By Theorem 1.5, it suffices to show that $\bar{g}$ satisfies (u5). Let $X \in \mathscr{P}(\mathscr{I})$ and let $I \in \bar{g}(X)$. Then $I \in \mathscr{P}_{X} \cup X$. Hence either $I \in \mathscr{P}_{X}$ or $I \in X$. If $I \in X$, then $I \in \bar{g}(\{I\})$ and $\{I\} \subseteq X$. If $I \in \mathscr{P}_{X}$, then $\exists J \in X$ such that $I \supseteq J$ and $I$ is a prime ideal. Hence $I \in \mathscr{P}_{\{J\}} \subseteq \bar{g}(\{J\})$ and $\{J\} \subseteq X$.

Define $g: \mathscr{P}(\mathscr{I}) \rightarrow \mathscr{P}(\mathscr{I})$ by $\forall X \in \mathscr{P}(\mathscr{I}), \underline{g}(X)=$ $\{I \in \mathscr{I} \mid \bar{g} \overline{(\{} I\}) \subseteq X\}$.

Theorem 2.8. Let $R$ be a commutative ring with identity and let $g$ be defined as above, where $V=\mathscr{I}$. Then $\underline{g}$ satisfies (11)-(14).

Proof. That $g$ satisfies (11) and (12) is immediate from the definition of $g$. Let $X, Y \subseteq \mathscr{P}(\mathscr{I})$. Then $J \in g(X) \cap g(Y) \Leftrightarrow J \in g(X)$ and $J \in g(Y) \Leftrightarrow \mathscr{P}_{\{J\}} \cup$ $\{J\} \subseteq X$ and $\mathscr{P}_{\{J\}} \cup\left\{\overline{J\}} \subseteq Y \Leftrightarrow \mathscr{P}_{\{J\}} \cup\{J\} \subseteq X \cap Y\right.$ $\Leftrightarrow J \in \underline{g}(X \cap Y)$. Hence $\underline{g}$ satisfies (13). Now $\underline{g}(X)$ is the union of those $\mathscr{P}_{\{I\}} \cup\{I\}$ which are contained in $X$ since $P \in \mathscr{P}_{\{I\}}$ and $\mathscr{P}_{\{I\}} \cup\{I\} \subseteq X \Rightarrow \mathscr{P}_{\{P\}} \cup\{P\}$ $\subseteq X$ and so $P \in \underline{g}(\{X\})$. Hence $\mathscr{P}_{\{I\}} \cup\{I\} \subseteq X$ implies $\mathscr{P}_{\{I\}} \cup\{I\} \subseteq \underline{g}(X)$. Hence $g(X) \subseteq g(g(X))$. However $g(g(X)) \subseteq \underline{g}(X)$ by (11) and (12). Thus (14) holds.
Let $X \in \mathscr{P}(\mathscr{I})$. Let $\mathscr{M}_{X}=\{M \in \mathscr{I} \mid \exists I \in X, M \supseteq I$, and $M$ is maximal $\}$. Let $\mathscr{M}=\{M \in \mathscr{I} \mid M$ is a maximal ideal\}.

Example 2.9. Let $R$ denote the polynomial ring $F[x, y]$ in algebraically independent indeterminates $x, y$ over the field $F$. Let $\bar{g}$ and $g$ be defined as above. Let $X=\mathscr{M}_{\{\langle x\rangle\}} \cup\{\langle x\rangle\}$. Then $\bar{g}(\{\langle x\rangle\})=X$, $\bar{g}(\{M\})=\{M\}, \forall M \in \mathscr{M}_{\{\langle x\rangle\}}$, and $\bar{g}(X)=X$ since a prime ideal strictly containing $\langle x\rangle$ is a maximal ideal of $R$. Thus, $g(X)=\{I \in \mathscr{I} \mid \bar{g}(\{I\}) \subseteq X\}=\left\{I \in \mathscr{I} \mid \mathscr{P}_{\{I\}}\right.$ $\left.\cup\{I\} \subseteq \mathscr{M}_{\{\langle x\rangle\}} \cup\{\langle x\rangle\}\right\}=X$ since either $I=\langle x\rangle$ or $I \in \mathscr{M}_{\{\langle x\rangle\}}$. Note that $\langle x\rangle$ is not a maximal ideal of $R$.

Example 2.10. Let $R, \bar{g}$ and $g$ be defined as in Example 2.9. Let $X=\left\{\langle x, y\rangle,\langle\bar{x}\rangle,\left\langle x^{2}\right\rangle\right\}$. Then $\bar{g}(X)$ $=\mathscr{P}_{X} \cup X=\mathscr{M}_{X} \cup\left\{\langle x\rangle,\left\langle x^{2}\right\rangle\right\}$. Now $g(X)=\{I \in \mathscr{I} \mid$ $\bar{g}(\{I\}) \subseteq X\}$. We have that $\bar{g}(\{\langle x\rangle\})=\mathscr{M}_{X} \cup\{\langle x\rangle\}$ $\nsubseteq X$ and $\bar{g}\left(\left\{\left\langle x^{2}\right\rangle\right\}\right)=\mathscr{M}_{X} \cup\left\{\langle x\rangle,\left\langle x^{2}\right\rangle\right\} \nsubseteq X$. However, $\bar{g}(\{\langle x, y\rangle\})=\{\langle x, y\rangle\} \subseteq X$. Hence $g(X)=$ $\{\langle x, y\rangle\} \subseteq X$.

Theorem 2.11. Let $R$ be a commutative ring with identity and $g$ be defined as above. Then $\forall I \in \mathscr{I}, g(\{I\}) \neq \emptyset$ if and only if $I$ is a maximal ideal of $R$.

Proof. $g(\{I\})=\{J \in \mathscr{I} \mid \bar{g}(J) \subseteq\{I\}\}=\left\{J \in \mathscr{I} \mid \mathscr{P}_{\{J\}}\right.$ $\cup\{J\} \subseteq\{I\}\}$. Thus either $g(\{I\})=\emptyset$ or $\exists J \in \mathscr{I}$ such that $\mathscr{P}_{\{J\}} \cup\{J\} \subseteq\{I\}$. If such a $J$ exists, then $J=I$ and $\mathscr{P}_{\{I\}}=\{I\} .\left(\mathscr{P}_{\{I\}} \neq \emptyset\right.$ since $I$ is contained in a maximal ideal.) Hence if such a $J$ exists, $J=I$ is a maximal ideal of $R$. That is, $\underline{g}(\{I\}) \neq \emptyset$ implies $I$ is a maximal ideal of $R$. Conversely, suppose $I$ is a maximal ideal of $R$. Then $\mathscr{P}_{\{I\}} \cup\{I\}=\{I\}$ and so $g(\{I\})=\{I\}$. Hence $g(\{I\}) \neq \emptyset$.

Corollary 2.12. Let $I \in \mathscr{I}$. Then the following assertions are equivalent.

(1) $g(\{I\}) \neq \emptyset$.

(2) $I$ is a maximal ideal of $R$.

(3) $g(\{I\})=\{I\}$.

(4) $\overline{\bar{g}}(\{I\})=\{I\}$.

(5) $\underline{g}(\{I\})=\bar{g}(\{I\})$.

Proof. That (1) $\Leftrightarrow$ (2) $\Leftrightarrow$ (3) follows from Theorem 2.11 and its proof. Now $g(\{I\})=\{I\} \Leftrightarrow \mathscr{P}_{\{I\}}$ $\cup\{I\} \subseteq\{I\} \Leftrightarrow \bar{g}(\{I\}) \subseteq\{I\} \Leftrightarrow \bar{g}(\{I\})=\{I\}$. Hence 
(3) and (4) are equivalent. Also $g(\{I\})=\bar{g}(\{I\}) \Leftrightarrow$ $\underline{g}(\{I\})=\mathscr{P}_{\{I\}} \cup\{I\} \Leftrightarrow \mathscr{P}_{\{I\}} \subseteq\{I\} \Leftrightarrow I$ is maximal. Hence (5) and (2) are equivalent.

Recall that $\mathscr{M}$ denotes the set of all maximal ideals of $R$. Then

$\bar{g}(\mathscr{M})=\bigcup_{M \in \mathscr{M}} \bar{g}(\{M\})=\bigcup_{M \in \mathscr{M}}\{M\}=\mathscr{M} \supseteq \underline{g}(\mathscr{M})$.

Now $\quad g(\mathscr{M})=\{J \in \mathscr{I} \mid \bar{g}(\{J\}) \subseteq \mathscr{M}\}=\mathscr{M} . \quad$ Thus $\bar{g}(\mathscr{M})=\underline{g}(\mathscr{M})=\mathscr{M}$.

Example 2.13. Let $R, \bar{g}$, and $g$ be defined as in Example 2.9. Let $X=\{\langle x\rangle\}$. Then $\langle x, y\rangle \in \mathscr{P}_{\{\langle x\rangle\}}$. Thus $\mathscr{P}_{\{\langle x\rangle\}} \cup\{\langle x\rangle\} \nsubseteq X$. Hence $\underline{g}(\{\langle x\rangle\})=\emptyset$.

We now determine structural results for $\mathscr{I}$ using the above concepts. Let $\mathscr{N}$ denote the set of all ideals of $R$ which are not prime. Then $\mathscr{I}=\bigcup_{J \in \mathscr{N}}\left(\mathscr{P}_{\{J\}} \cup\{J\}\right)=\bigcup_{J \in \mathscr{N}} \bar{g}(\{J\})=\bar{g}(\mathscr{N})$. Hence $\mathscr{N}$ generates $\mathscr{I}$ (with respect to $\bar{g}$ ). Let $I \in \mathscr{N}$. Then

$$
I \notin \bigcup_{J \in \mathscr{N} \backslash\{I\}}\left(\mathscr{P}_{\{J\}} \cup\{J\}\right)=\bar{g}(\mathscr{N} \backslash\{I\}) .
$$

Thus $\mathscr{N}$ is a basis for $\mathscr{I}$ (with respect to $\bar{g}$ ).

Now $\bar{g}(\{I\})=\bar{g}(\bar{g}(\{I\}))$ by Theorem 2.7. If $I, J \in \mathscr{N}$ and $I \neq J$, then $\bar{g}(\{I\}) \subseteq \bar{g}(\{J\})$ is impossible since $I \notin \bar{g}(\{J\})$. Thus $\{\bar{g}(\{I\}) \mid I \in \mathscr{N}\}$ is the set of primaries of $\mathscr{I}$ [7, p. 179], and $\mathscr{I}=\bigcup_{I \in \mathcal{N}} \bar{g}(\{I\})$. However, we see that this union is not a direct union, i.e., it is not the case that, in general $\bar{g}(\{I\}) \cap \bar{g}(\{J\})=\emptyset$ when $\bar{g}(\{I\}) \neq \bar{g}(\{J\})$ and $I, J \in \mathscr{N}$.

\section{Fuzzy ideals}

We now examine the situation for fuzzy ideals of a commutative ring with identity $R$. Let $L$ be a complete distributive lattice. An $L$-subset of $R$ is a function of $R$ into $L$. If $S$ is a subset of $R$ and $a \in L$, we define the fuzzy subset $a_{S}$ of $R$ by $\forall x \in R, a_{S}(x)=a$ if $x \in S$ and $a_{S}(x)=0$ otherwise. We use the notion of the $L$-prime spectrum of $R[10,1-5]$, to define a cover of $\mathscr{F} \mathscr{I}=\left\{I \mid I\right.$ is an $L$-ideal of $\left.R, I \neq 1_{R}\right\}$. Let $\mathscr{F} \mathscr{P}$ denote the set of all prime $L$-ideals of $R$ and $\forall X \in \mathscr{P}(\mathscr{F} \mathscr{I})$, the $L$-power set of $\mathscr{F} \mathscr{I}$, let
$\mathscr{F} \mathscr{P}_{X}$ denote $\{P \in \mathscr{F} \mathscr{P} \mid \exists I \in X$ such that $P \supseteq I\}$. Then $\mathscr{F} \mathscr{P}$ is known as the $L$-prime spectrum of $R$ and the closed subsets of $\mathscr{F} \mathscr{P}$ in the Zariski topology are exactly the $\mathscr{F} \mathscr{P}_{\{I\}}$, where $I \in \mathscr{F} \mathscr{I}$. Let $\mathscr{C}=\left\{\mathscr{F} \mathscr{P}_{\{I\}} \cup\{I\} \mid I \in \mathscr{F} \mathscr{I}\right\}$. Then $\mathscr{C}$ is a cover of $\mathscr{F} \mathscr{I}$. If $I$ is an $L$-ideal of $R$, then $I_{*}=\{x \in R \mid I(x)=$ $I(0)\}$ is an ideal of $R$.

Define $\bar{s}: \mathscr{P}(\mathscr{F} \mathscr{I}) \rightarrow \mathscr{P}(\mathscr{F} \mathscr{I})$ as in Definition 1.2, i.e., $\forall X \in \mathscr{P}(\mathscr{F} \mathscr{I}), \bar{s}(X)=\left\{J \in \mathscr{F} \mathscr{I} \mid \exists \mathscr{F} \mathscr{P}_{\{I\}} \cup\{I\}\right.$ $\in \mathscr{C}, J \in \mathscr{F}_{\{I\}} \cup\{I\}$ and $\left.\left(\mathscr{F}_{\{I\}} \cup\{I\}\right) \cap X \neq \emptyset\right\}$. Define $\quad \bar{g}: \mathscr{P}(\mathscr{F} \mathscr{I}) \rightarrow \mathscr{P}(\mathscr{F} \mathscr{I}) \quad$ by $\quad \forall X \in \mathscr{P}(\mathscr{F} \mathscr{I})$, $\bar{g}(X)=\mathscr{F} \mathscr{P}_{X} \cup X$. Then $\mathscr{C}$ is the cover induced by $\bar{g}$. In fact, $\bar{g}$ here defines $\mathscr{C}$ just as does the $\bar{g}$ in Section 1 since $\mathscr{C}=\{\bar{g}(\{I\}) \mid I \in \mathscr{F} \mathscr{I}\}$. Define $\quad g: \mathscr{P}(\mathscr{F} \mathscr{I}) \rightarrow \mathscr{P}(\mathscr{F} \mathscr{I}) \quad$ by $\quad \forall X \in \mathscr{P}(\mathscr{F} \mathscr{I})$, $\underline{g}(X)=\{\bar{I} \in \mathscr{F} \mathscr{I} \mid \bar{g}(\{I\}) \subseteq X\}$. An $L$-ideal $M$ of $R$ is called maximal if $M \neq 1_{R}$ and there is no $L$-ideal of $R$ strictly containing $M$ other than $1_{R}$. Then an $L$-deal of $R$ is maximal if and only if $M$ can be expressed as $M=1_{M_{*}} \cup a_{R}$, where $a \in L \backslash\{0\}$ is a dual atom (maximal element) of $L$ and $M_{*}$ is a maximal ideal of $R$. Now the results in Section 2 can be extended immediately to $L$-ideals except for those dealing with maximal ideals. For example, let $L=[0,1]$. Then $L$ has not dual atoms and so $R$ has no maximal $L$-ideals in the usual sense. Hence Theorem 2.11 and Corollary 2.12 cannot be immediately extended to $L$-ideals.

We now provide some details to the discussion of the extension of the results of Section 2 to $L$-ideals. The proofs of the following results are the same as for crisp ideals.

Theorem 3.1. Let $R$ be a commutative ring with identity and let $\bar{s}, \bar{g}$, and $g$ be defined as above. Then the following assertion hold:

(1) $\forall X \in \mathscr{P}(\mathscr{F} \mathscr{I}), \bar{g}(X) \subseteq \bar{s}(X)$.

(2) If $K$ is an $L$-ideal of $R$ which is not prime, then $\bar{s}(\{K\})=\mathscr{F}_{\{K\}} \cup\{K\}$.

(3) If $P$ is a prime L-ideal, then $\bar{s}(\{P\})=\mathscr{F} \mathscr{P} \cup S_{P}$, where $S_{P}=\{I \in \mathscr{F} \mathscr{I} \mid I \subseteq P\}$.

(4) Then $\bar{g}$ satisfies (u1)-(u4) and (u6).

(5) Then g satisfies (11)-(14).

Proposition 3.2. Let $R$ be a quasi-local ring. Let $\bar{s}$ be defined as above. Suppose that L has a unique dual atom, e.g., $L$ is a finite chain. Let $M$ denote the unique maximal $L$-ideal of $R$. Then $\bar{s}(\{M\})=\mathscr{F} \mathscr{I}$. 
We recall that an $L$-ideal of $R$ is called a generalized maximal $L$-ideal if for any $L$-ideal $J$ of $R, I \subseteq J$ implies that either $I_{*}=J_{*}$ or $J=1_{R}$. Then a generalized maximal $L$-ideal of $R$ can be characterized as follows: An $L$-ideal $I$ of $R$ is a generalized maximal ideal if and only if $I$ can be expressed as $I=1_{I_{*}} \cup a_{R}$, where $I_{*}$ is a maximal ideal of $R$ and $a \in L \backslash\{1\}$ [13, p. 97].

Suppose that $L=[0,1]$. Let $I \in \mathscr{F} \mathscr{I}$ be a generalized maximal $L$-deal. Then $\mathscr{F} \mathscr{P}_{\{I\}} \cup\{I\}=\{J \mid J \supseteq I$, $J$ is a generalized maximal $L$-ideal $\}$ and it is impossible for $\mathscr{F} \mathscr{P}_{\{I\}} \cup\{I\} \subseteq\{I\}$ since $I$ has an infinite number of generalized maximal $L$-ideals which contain $I$. (Any generalized maximal $L$-ideal $J$ of $R$ with $J_{*}=I_{*}$ and $J(x)>I(x) \forall x \in R \backslash I_{*}$ is a member of $\mathscr{F}_{\{I\}}$.) Hence Theorem 2.11 and Corollary 2.12 are not immediately extendible. However, we do have the following extensions.

Theorem 3.3. Suppose that every element of $L \backslash\{1\}$ is contained in a dual atom. Let $R$ be a commutative ring with identity and $g$ be defined as above. Then $\forall I \in \mathscr{F} \mathscr{I}, \underline{g}(\{I\}) \neq \emptyset$ if and only if $I$ is a maximal L-ideal of $\bar{R}$.

Corollary 3.4. Suppose that every element of $L \backslash\{1\}$ is contained in a dual atom. Let $I \in \mathscr{F} \mathscr{I}$. Then the following assertions are equivalent.

(1) $\underline{g}(\{I\}) \neq \emptyset$.

(2) $\bar{I}$ is a maximal L-ideal of $R$.

(3) $\underline{g}(\{I\})=\{I\}$.

(4) $\overline{\bar{g}}(\{I\})=\{I\}$.

(5) $\underline{g}(\{I\})=\bar{g}(\{I\})$.

For further research projects, one might examine Theorem 2.11 and Corollary 2.12 for $L=[0,1]$ since $L$ in this case is the lattice most often used and for which most of the applications exist. Also, we know that the closed subsets of $\mathscr{F} \mathscr{P}$ in the Zariski topology are exactly the $\mathscr{F} \mathscr{P}_{\{I\}}$, where $I \in \mathscr{F} \mathscr{I}$. Because of the importance of the $\mathscr{F}_{\{I\}} \cup\{I\}$ in the above development, their topological properties may be of interest. The ultimate goal is to further develop the ideas initiated here and apply them to the study of fuzzy intersection equations. A possible start is to examine the examples of fuzzy intersection equations given and studied in [13].

\section{References}

[1] H.H. Abadi, M.M. Zahedi, Some results on fuzzy prime spectrum of a ring, Fuzzy Sets and Systems 77 (1996) 235-240.

[2] J.K. Kohli, R. Kumar, Fuzzy prime spectrum of a ring II, Fuzzy Sets and Systems 59 (1993) 223-230.

[3] R. Kumar, Fuzzy prime spectrum of a ring, Fuzzy Sets and Systems 46 (1992) 147-154.

[4] H.V. Kumbhojkar, Spectrum of prime fuzzy ideals, Fuzzy Sets and Systems 62 (1994) 101-109.

[5] H.V. Kumbhojkar, Some comments on spectrums of prime fuzzy ideals of a ring, Fuzzy Sets and Systems 85 (1997) 109-114.

[6] E. Kunz, Introduction to Commutative Algebra, Birkhauser, Basel, 1985.

[7] N. Kuroki, J.N. Mordeson, Successor and source functions, J. Fuzzy Math. 5 (1997) 173-182.

[8] N. Kuroki, J.N. Mordeson, Structure of rough sets and rough groups, J. Fuzzy Math. 5 (1997) 183-191.

[9] D.S. Malik, J.N. Mordeson, Fuzzy prime ideals of a ring, Fuzzy Sets and Systems 37 (1990) 93-98.

[10] D.S. Malik, J.N. Mordeson, $L$-prime spectrum of a ring, Proc. NAFIPS'97 97TH8297, 1997, pp. 273-278.

[11] J.N. Mordeson, Fuzzy algebraic varieties, Rocky Mountain, J. Math. 23 (1993) 1361-1377.

[12] J.N. Mordeson, Fuzzy algebraic varieties II, in: P.P. Wang (Ed.), Advances in Fuzzy Theory and Technology, vol. I, Bookswright Press, Durham NC, 1995, pp. 9-21.

[13] J.N. Mordeson, D.S. Malik, Fuzzy Commutative Algebra, World Scientific, Singapore, 1998.

[14] J.N. Mordeson, P.S. Nair, Retrievability and connectedness in fuzzy finite state machines, Proceedings of the 5th IEEE Internat. Conf. Fuzzy Systems, vol. 3, 1996, pp. 1586-1590.

[15] J.N. Mordeson, P.S. Nair, Connectedness in systems theory, Proceedings of the 5th IEEE Internat. Conf. on Fuzzy Systems, vol. 3, 1996, pp. 2045-2048.

[16] T.K. Mukherjee, M.K. Sen, On fuzzy ideals of a ring I, Fuzzy Sets and Systems 21 (1987) 99-104.

[17] E. Orlowska, Semantic analysis of inductive reasoning, Theoret. Comput. Sci. 43 (1986) 81-89.

[18] Z. Pawlak, Rough sets, Internat. J. Comput. Sci. 11 (1982) 341-356.

[19] Z. Pawlak, Rough Sets, Theoretical Aspects about Data, Kluwer Academic Pub, Dordrecht, 1991.

[20] J.A. Pomykala, Approximation operations in approximation space, Bull. Polish Acad. Sci. Math. 35 (1987) 653-662.

[21] A. Rosenfeld, Fuzzy groups, J. Math. Anal. Appl. 35 (1971) 512-571.

[22] R. Slowinski, D. Vanderpooten, Similarity relation as a basis for rough approximations, in: P.P. Wang (Ed.), Advances in Machine Intelligence \& Soft-Computing, vol. IV, Department of Electrical Engineering, Duke University, Durham, North Carolina, USA, 1997, pp. 17-33.

[23] U.M. Swamy, K.L.N. Swamy, Fuzzy prime ideals of a ring, J. Math. Anal. Appl. 134 (1988) 94-103. 
[24] A. Wasilewska, Conditional knowledge representation systems - model for an implementation, Bull. Polish Acad. Sci. Math. 37 (1987) 63-69.

[25] A. Wasilewska, L. Vigneron, On generalized rough sets, in: P.P. Wang (Ed.), Proc. 3rd Joint Conf. on Inf. Sci., vol. 3, 1997, pp. $165-168$.

[26] U. Wybraniec-Skardowska, On a generalization of approximation space, Bull. Polish Acad. Sci. Math. 37 (1989) 51-61.

[27] Y.Y. Yao, Relational interpretations of neighborhood operators and rough set approximation operators, Inf. Sci. 111 (1998) 239-259.
[28] Y.Y. Yao, Two views of the theory of rough sets in finite universes, Internat. J. Approx. Reason. 15 (1996) 291-317.

[29] Y.Y. Yao, T.Y. Lin, Generalization of rough sets using modal logics, Intelligent Automat. Soft Comput. 2 (1996) $103-120$.

[30] L.A. Zadeh, Fuzzy sets, Inform. and Control 8 (1965) 338-353.

[31] W. Zakowski, Approximations in the space (U, II), Demonstratio Math. XVI (1983) 761-769.

[32] O. Zariski, P. Samuel, Commutative Algebra, vol. I, D. Van Nostrand Company, Inc., Princeton, NJ, 1958. 